\title{
CHANGES IN PHYSIOLOGICAL CHARACTERISTICS AND TRANSCRIPT EXPRESSION PROFILES IN THE LATENT BUD SPROUTING OF PINUS MASSONIANA (MASSON'S PINE)
}

\author{
ZHU, Y. Y. $.^{1 \#}-$ QI, D. P. ${ }^{2 \#}-$ ZHAO, Y. ${ }^{3,4}-$ XIAO, F. ${ }^{3}-$ YANG, B. ${ }^{1}-$ XU, J. J. ${ }^{1}-$ ZHOU, J. W. ${ }^{1}-$ WANG, G. ${ }^{1 *}$ \\ ${ }^{1}$ Guizhou Academy of Forestry, Guiyang, Guizhou, China \\ ${ }^{2}$ Guizhou Meteorological Center, Guiyang, Guizhou, China \\ ${ }^{3}$ Guizhou University, Guiyang, Guizhou, China
}

${ }^{4}$ Guizhou Provincial Forest Resources and Environment Research Center, Guiyang, Guizhou, China

*Corresponding author

e-mail: zyynjfu@163.com; phone: +86-182-7537-4373

${ }^{\#}$ These authors have contributed equally to this work and should be considered as co-first authors

(Received $15^{\text {th }}$ Oct 2020; accepted 21 ${ }^{\text {st }}$ Dec 2020)

\begin{abstract}
The latent bud regeneration ability at the center of Pinus massoniana needle bundles is strong, and can provide a large amount of material for asexual reproduction. Topping $P$. massoniana can induce latent bud sprouting, but the mechanisms underlying the sprouting process are unclear. In this study, physiological indexes were examined during the sprouting of coniferous buds. Additionally, the transcript expression profile of the sprouting process was obtained by high-throughput sequencing. The results showed that increases in $\mathrm{N}$ and the endogenous hormone indole-3-acetic acid (IAA) could promote dormancy release and lateral branching of $P$. massoniana. Abscisic acid had a protective effect on the dormancy of $P$. massoniana buds. The expression of phenylpropanoid biosynthesis- and flavonoid biosynthesis-related genes may be induced by differences in test material synthesis and transport caused by changes in the nutrient composition of $P$. massoniana needles after apical topping. The observed upregulated expression of IAA transport- and response-related genes may be pertinent to the dormancy release of axillary buds. The observed activation of transcription factors within the TCP and MADS-box families may be associated with lateral meristem formation. The results of this study provide fundamental data for studying the dormancy and development of latent buds in $P$. massoniana.
\end{abstract}

Keywords: Pinus massoniana, latent bud, dormancy release, endogenous hormone, topping

Abbreviations: IAA: indole-3-acetic acid; $\mathrm{GA}_{3}$ : gibberellic acid; ABA: abscisic acid; ZR: zeatin riboside; POD: peroxidase; 6-BA: 6-benzylaminopurine; SOD: superoxide dismutase; TCP: TCP family transcription factor; MADS-box: MADS-box gene family; NBT: nitroblue tetrazolium; CTAB: cetyl trimethylammonium bromide; TPM: transcripts per million; TMM: trimmed mean of M-values; GO: gene ontology; DEGs: differentially expressed genes; KEGG: The Kyoto Encyclopedia of Genes and Genomes

\section{Introduction}

The needles of Pinus massoniana are extremely shortened shoots. The latent buds at the center of the base of the needle bundles can develop, grow and form new shoots under certain conditions. This regenerative ability is very strong and can provide a large amount of material for the asexual reproduction of $P$. massoniana, which can accelerate the breeding process. Previous study of the growth process of the latent buds of $P$. massoniana has shown that the effect of temperature on the sprouting of latent buds is the most direct. It has also been found that the time required between pruning and sprouting is the longest in winter, and the shortest in summer (only about $23 \mathrm{~d}$ ). The 
sprouting rate was negatively correlated with the age of the tree. The average number, length and diameter of latent buds grown with different concentrations of the exogenous hormones indole-3-acetic acid (IAA), gibberellic acid $\left(\mathrm{GA}_{3}\right)$ and 6-benzylaminopurine (6-BA) were higher than those of the untreated control buds (Zhu et al., 2018).

There have been many reports on the application of coniferous latent bud sprouting in pines used for production. Chen et al. (1989) used coniferous bundles of young shoots of $P$. elliottii, $P$. taeda and $P$. massoniana as scions, and successfully curated a set of grafting techniques that use the coniferous latent buds to sprout into branches. A regeneration system for $P$. massoniana using the axillary buds at the base of the coniferous leaves of robust seedlings was by established (Zhang et al., 2006; Zhu et al., 2010). At present, the cause and mechanism of latent bud sprouting are not clear. Transcriptome (RNA sequencing) studies are essential for identifying functional elements of the genome, revealing the molecular components of cells and tissues, and determining development. In this study, changes in endogenous hormone content, peroxidase (POD) and superoxide dismutase (SOD) activities and differential expression of endogenous substances involved in the N, P, K, soluble sugar, starch and IAA pathways were investigated during the sprouting of coniferous latent buds. The results provided fundamental theoretical data for the relaxation and development of latent bud dormancy in P. massoniana.

\section{Materials and methods}

\section{Materials}

The test materials were taken from the $P$. massoniana seed orchard of the Guizhou Academy of Forestry, China. Twelve healthy 6-year-old P. massoniana plants of similar growth and with no pests or diseases were selected. The apical tips were trimmed to disrupt the apical dominance. The sampling technique was as described by $\mathrm{Xu}$ et al. (1992), who used the technique for researching larch needle nutrition. The sampling was carried out at approximately $10 \mathrm{am}$ in the morning and the nutrient content of the needles was stable. Physiological indicators were sampled every 5 days until the latent buds germinated. Four sample groups, including the control (labeled P1) were used in this study. The needle sheaths were removed from the plants in all groups. In the control group (P1), the pine plant was not trimmed. In the group labeled P2, the needle bundle bases of the main branch and the side branch, and the phloem joint were trimmed $10 \mathrm{~d}$ after trimming the apical tips. In the group labeled P3, these tissues were trimmed after $20 \mathrm{~d}$. In the group labeled P4, these tissues were trimmed after $30 \mathrm{~d}$, by which point the axillary buds had sprouted. The transcriptomic samples were obtained for each of these groups after mixing and registering.

\section{Methods}

\section{Determination of physiological indicators}

The content of hormones such as IAA and ABA were determined using enzymelinked immunosorbent assays. The soluble sugar and starch contents were determined using the anthrone colorimetric method as described by Wang (2006). The activity of SOD was determined using nitroblue tetrazolium (NBT) reduction. The activity of POD was determined using guaiacol. The $\mathrm{N}$ content was determined using the Kjeldahl method and the $\mathrm{P}$ content was determined using the vanadium molybdate yellow 
colorimetric method. The $\mathrm{K}$ content was determined using atomic absorption spectrophotometry. Visualization and inter-group difference analysis was performed using the R package ggstatsplot (Patil, 2018).

\section{RNA extraction and library preparation}

The RNA was extracted and purified using the CTAB, and the RNA quality and concentration were tested $(\mathrm{A} 260 / \mathrm{A} 280=2.0-2.2 ; \quad \mathrm{A} 260 / \mathrm{A} 230=1.8-2.2$; $28 \mathrm{~S} / 18 \mathrm{~S}=1.4-2.7$; RNA integrity number $[\mathrm{RIN}] \geq 8.0$ ). The quality of the constructed library was determined using an Agilent 2100 Bioanalyzer. The library was sequenced using an Illumina HiSeq X Ten sequencer to generate $150 \mathrm{bp}$ double-ended data.

\section{Evaluation, assembly and annotation of raw data quality}

Trimmomatic was used to filter raw reads and remove the low-quality sequences with linkers (Bolger et al., 2014). Using Trinity (Haas et al., 2013), de novo assembly of transcripts was performed based on the clean data, and then redundant contigs were removed. The longest transcript in each transcript cluster was taken as a unigene, and unigenes were compared with multiple databases such as eggNOG, NR, NT, GO, Pfam, Swiss-Pro.

Gene expression quantification, differential expression analysis and functional enrichment

The spliced transcript was used as a reference sequence, and the quality-controlled assembled sequences were aligned with the reference sequence using Bowtie 2 (Langmead et al., 2012). The results were statistically compared using RSeQC. The TPM (Transcripts Per Million) calculation was performed in conjunction with Salmon software (Patro et al., 2017). Correlation heat maps were constructed using the different gene expression levels between samples. The number of shared and uniquely expressed genes $($ TPM $>0)$ in the samples were counted by Venn diagram. The read count data was normalized using TMM, and DEGseq was used to identify differentially expressed genes from the data without biological replicates. The conditions used for screening for differential gene expression were qValue $<0.05$ and $\mid$ FoldChange $\mid>2$. Gene expression clustering was performed on genes that displayed significant differences in expression between the experimental groups, using the $\mathrm{R}$ package gplots. Functional enrichment analysis of GO and KEGG enrichment were performed using Cluster Profiler ( $\mathrm{Yu}$ et al., 2012). Visualization of gene annotation and expression was performed using MapMan (http://mapman.gabipd.org/). Real-time quantitative PCR (RT-qPCR) was performed on a real-time CFX96 Touch PCR instrument. The first strand of the cDNA fragment was synthesized from total RNA. The PCR reaction conditions were as follows: preheating at $95^{\circ} \mathrm{C}$ for $30 \mathrm{~s}, 40$ cycles of heat denaturation at $95^{\circ} \mathrm{C}$ for $5 \mathrm{~s}$, and annealing at $60^{\circ} \mathrm{C}$ for $34 \mathrm{~s}$.

\section{Results}

\section{External morphological changes during the development of coniferous latent buds}

The seeds of pine species sprout long leaves. First, the cotyledons grow, then the primary leaves grow at the top of the main stem, and finally the needles (secondary 
leaves) grow from the axil of the scale leaves. The first leaves of the strips are more original than secondary leaves. The primary leaves of the seedlings and the secondary leaves of the trees are very different, which leads to morphological variation between trees during their development. The seedling morphology is a reenactment of the original form of its ancestors, which is also known as the phenomenon of returning to the ancestors (Qin et al., 2012). Before the sprouting of the coniferous latent buds, the needles became thicker, with an average diameter of $0.52 \mathrm{~mm}(0.46 \mathrm{~mm}$ in the control group). As shown in Figure 1, the latent buds erupted from the base of the needle bundle and were flat (Fig. Ia). The strip-shaped leaves differentiated and separated (Fig. $1 b-f)$. The shoots were then ejected and normal needles erupted from the base (Fig. 1 g). This phenomenon indicates that the latent buds of $P$. massoniana have the potential to develop juvenile characteristics.
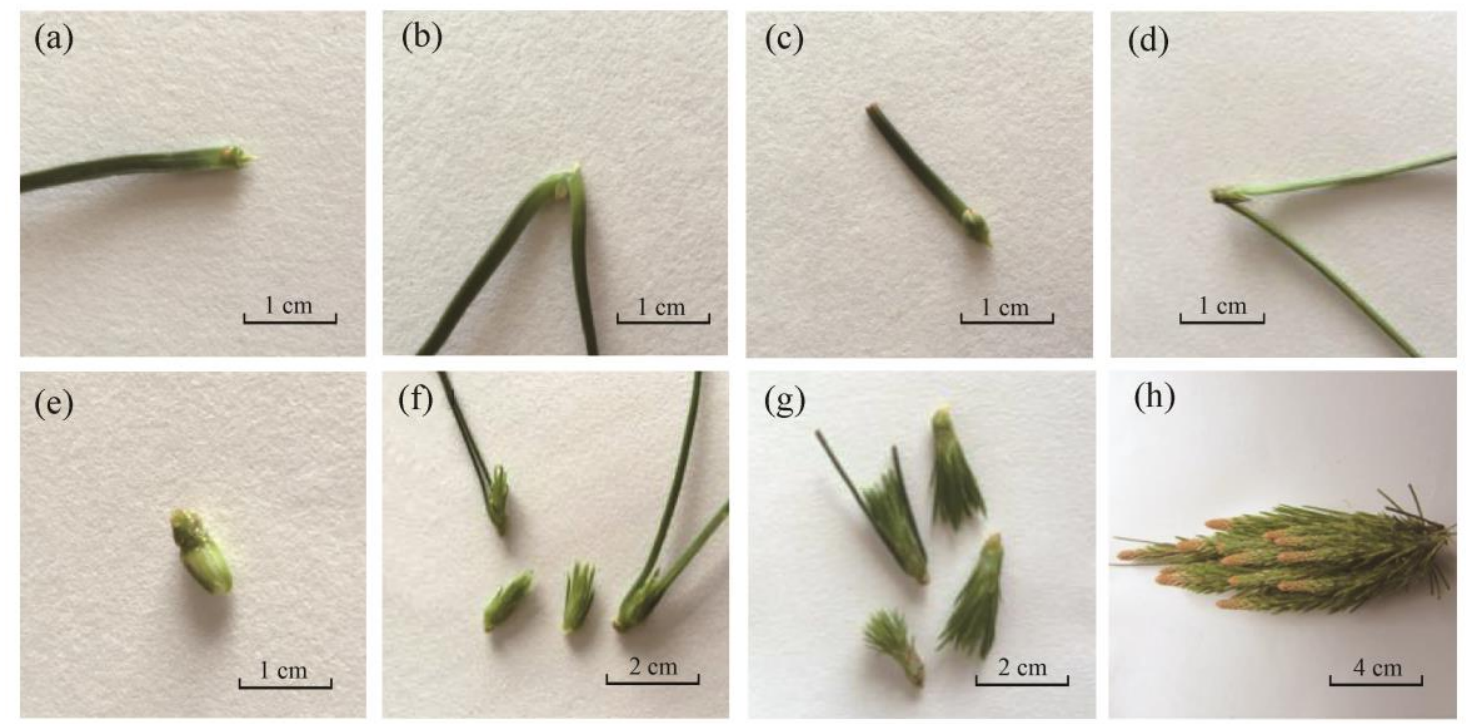

Figure 1. External morphological changes during the development of latent buds of $P$. massoniana

\section{Analysis of physiological indicators}

There were several physiological indicators that displayed significant differences between the experimental groups $(0 \mathrm{~d} ; 5 \mathrm{~d} ; 10 \mathrm{~d} ; 15 \mathrm{~d} ; 20 \mathrm{~d} ; 25 \mathrm{~d} ; 30 \mathrm{~d})$. These included $\mathrm{N}$ content, $\mathrm{GA}_{3}$ content, zeatin riboside (ZR) content, soluble sugar content and SOD activity ( $<<0.05 ;$ Fig. 2). The $\mathrm{N}$ content showed an increasing pattern from $0 \mathrm{~d}$ to $30 \mathrm{~d}$. The correlation between $\mathrm{N}$ and $\mathrm{K}$ content was 0.6 . When the apex dominance was broken (from $0 \mathrm{~d}$ to $5 \mathrm{~d}$ ), the ABA content decreased sharply, from $83.60 \mathrm{ng} / \mathrm{g}$ to $63.16 \mathrm{ng} / \mathrm{g}$, and then decreased steadily. The IAA content increased continuously from $50.90 \mathrm{ng} / \mathrm{g}$ to $82.16 \mathrm{ng} / \mathrm{g}$ at the peak, and after $20 \mathrm{~d}$, the content decreased slightly. IAA and $\mathrm{ABA}$ showed negative correlation, with a correlation coefficient of -0.48 . The $\mathrm{GA}_{3}$ content gradually increased, but decreased after $15 \mathrm{~d}$, and finally returned to its new balance. The ratio of (IAA $+\mathrm{GA}_{3}+\mathrm{ZR}$ )/ABA gradually increased from 0.796 to 1.746 at the time of bud sprouting, and the endogenous hormones reached a new equilibrium after sprouting. There was a 
significant positive correlation between the soluble sugar and total starch contents; the correlation coefficient was $0.68(\mathrm{r}>0.5)$.
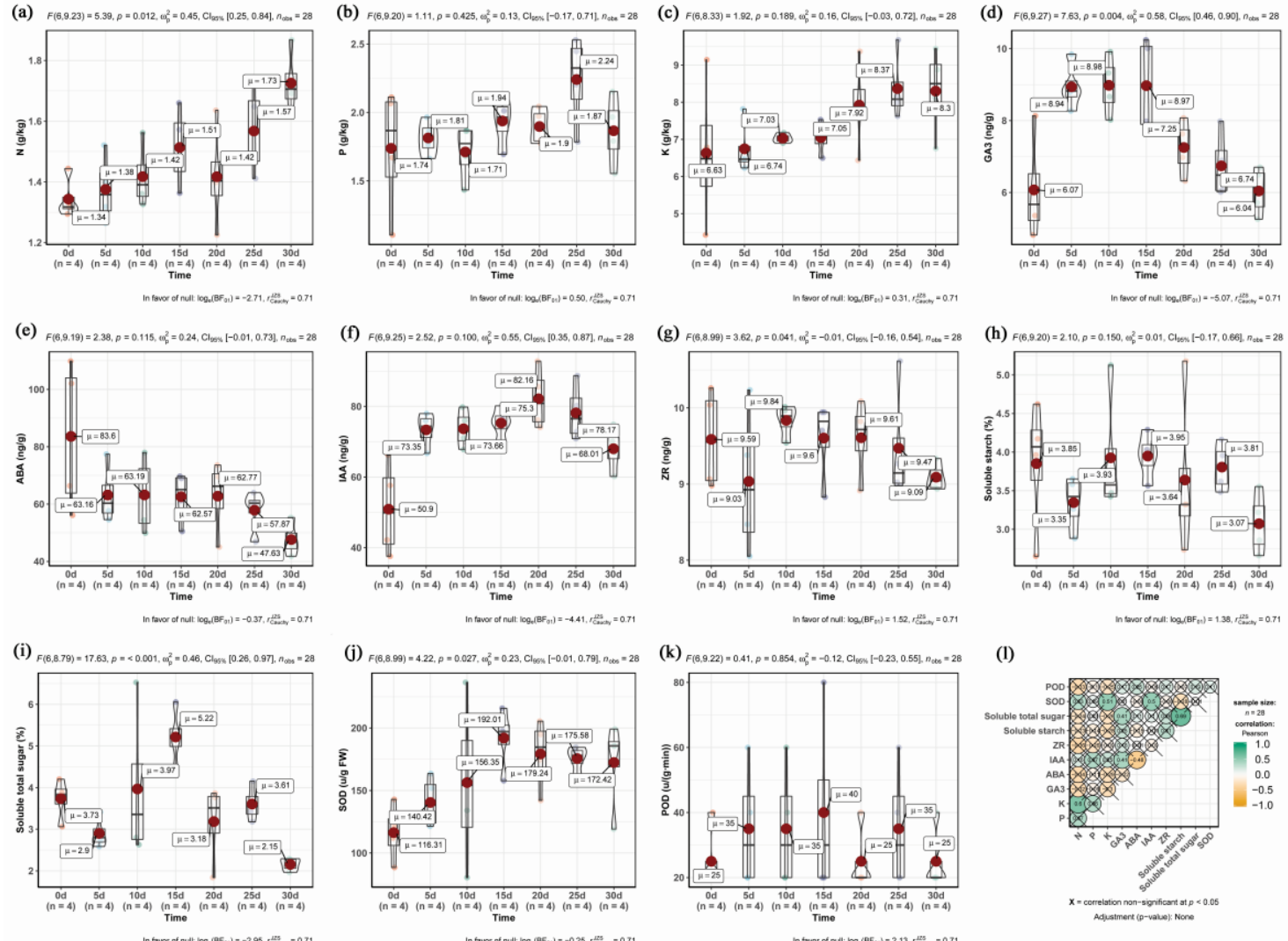

Figure 2. (a) $N$ content changes; (b) P element content changes; (c) K element content changes; (d) GA hormone content changes; (e) ABA hormone content changes; $(f)$ IAA hormone content changes; $(g)$ ZR hormone content changes; $(h)$ Changes of soluble starch content; $(i)$ change of soluble sugar content; $(j)$ change of SOD content; $(k)$ change of POD content; $(l)$ correlation between indicators

\section{Analysis of transcriptomic expression differences}

Data quality control, splicing, annotation and comparison

In terms of the statistics on raw data quality values, the needle bundle base mass values were well distributed and the distribution of the four sample (P1-P4) bases was uniform. The number of unigenes spliced was 169751, the N50 was $393 \mathrm{bp}$, the N90 was $221 \mathrm{bp}$, and the average length was $392.82 \mathrm{bp}$. The proportion of genes that were successfully annotated in at least one database accounted for $61.59 \%$ of the total number of genes. The highest rate of annotation in a single database was $48.55 \%$, which was observed in the Gene Ontology (GO) database. As a species that does not have a whole-genome sequence, the annotation ratio for $P$. massoniana was relatively high. The distribution frequency of redundant sequences from the samples showed that the overall trend was linear and smoothly extended; the content of redundant sequences was normal. Testing for the uniformity of distribution showed that the sequences were evenly distributed within the genes. 


$$
-732-
$$

\section{Correlation between samples and determination of differentially expressed genes}

The correlation coefficient between each sample was high (Fig. 3a). The Venn diagram (Fig. 3b) showed that the number of expressed genes (TPM $>0$ ) in the samples were highly similar. Inter-group differentially expressed genes (DEGs) were identified based on expression levels and statistical findings (Fig. 3c). There were 7349 DEGs between P2 and P1, of which 5798 were upregulated and 1551 were downregulated. There were 5407 DEGs between P3 and P1, of which 2345 were upregulated, and 3062 were downregulated. There were 12,519 DEGs between P4 and P1, of which 9489 were upregulated and 3030 were downregulated. There were 2284 DEGs that were shared among the three comparisons (Fig. 3d).

(a)

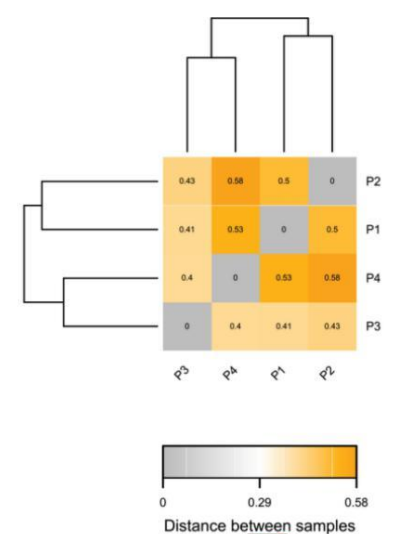

(c)

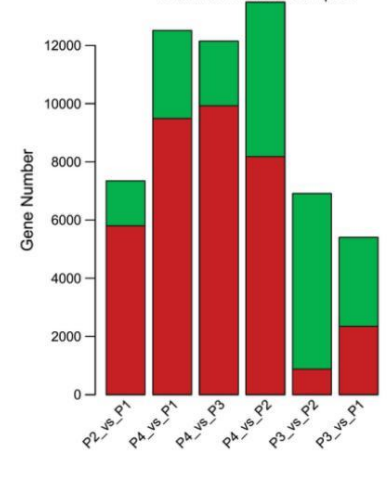

(b)

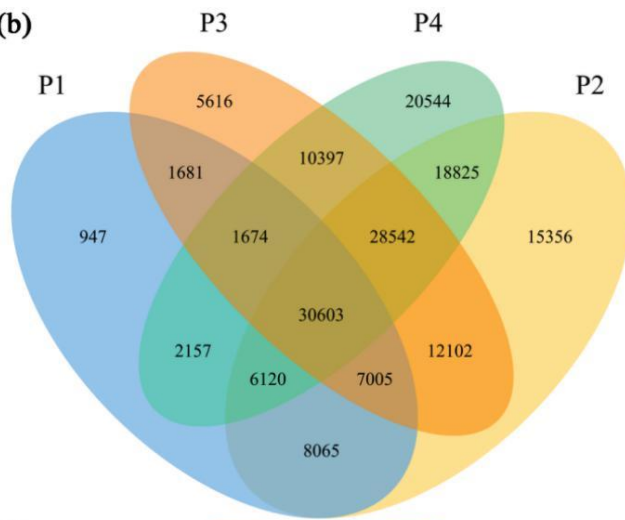

(d)

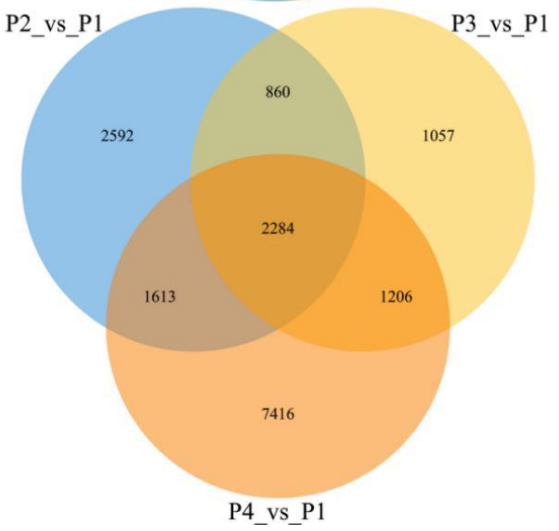

Figure 3. (a) Heat map of correlation analysis between samples; (b) Co-expression of Venen map; (c) Bar graph of differential gene numbers of two pairs; (d) Venen plot of differential gene between different combinations

\section{Differential gene trend clustering and functional enrichment}

The Kyoto Encyclopedia of Genes and Genomes (KEGG) enrichment analysis was performed on the significantly differentially expressed genes obtained from the three comparisons ( $\mathrm{P} 2$ vs $\mathrm{P} 1, \mathrm{P} 3$ vs $\mathrm{P} 1$ and $\mathrm{P} 4$ vs $\mathrm{P} 1$ ). The 10 pathways with the smallest $\mathrm{p}$ values were selected for multi-channel enrichment visualization. The phenylpropanoid biosynthesis (ko00940) and flavonoid biosynthesis (ko00941) pathways were enriched in the three different comparisons (Fig. 4a). In the category of biological processes, GO enrichment analysis of the DEGs in the P4 vs P1 group (Fig. 4b) found that responses to oxygen-containing compounds and responses to abiotic stimulus were enriched. Expression pattern cluster analysis of the DEGs of the four comparison groups revealed 


$$
-733 \text { - }
$$

that most DEGs (4499; subcluster 2) displayed increasing expression with time (Fig. 4c). GO enrichment analysis of subcluster 2 genes (Fig. 4d) found that in the biological processes category, the response to abiotic stimulus, and hormone and cellulose biosynthetic processes were enriched. KEGG enrichment analysis of subcluster 2 genes (Fig. 4e) found that among other pathways, protein processing in endoplasmic reticulum (ko04141), starch and sucrose metabolism (ko00500), and plant hormone signal transduction (ko04075) were enriched.

(a)

(b)
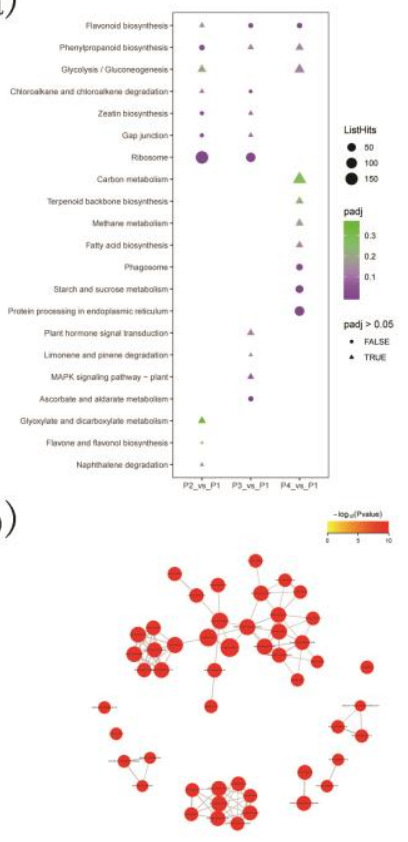

(c)
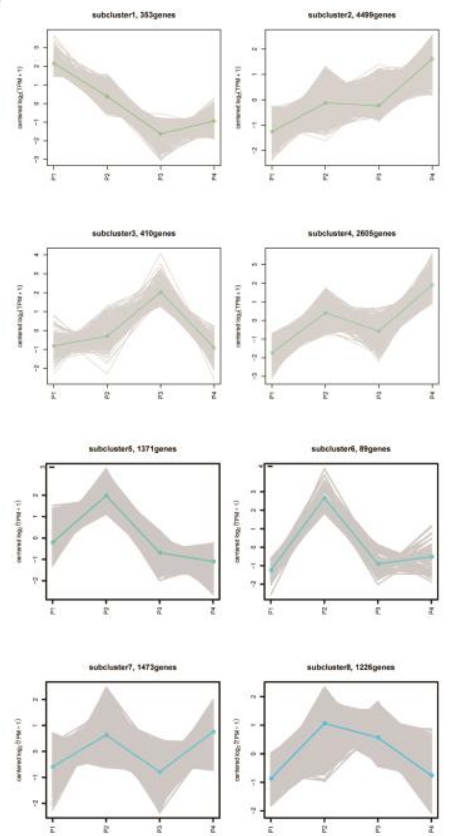

(d)

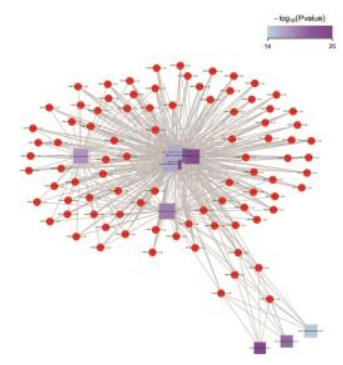

(e)

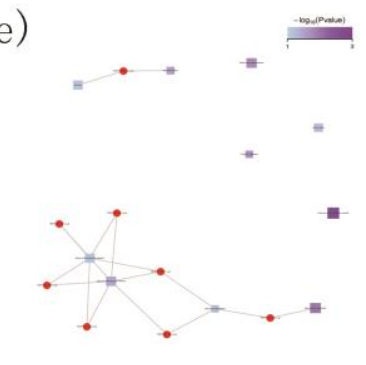

Figure 4. (a) multiple sets of KEGG pathway enrichment maps; (b) P4 vs Pl GO enrichment network maps (BP levels); (c) Trend line graph of differential gene expression; (d) GO enrichment analysis of subcluster2; (e) KEGG enrichment analysis of subcluster2

Among the plant hormone signaling pathways of subcluster 2, there were 18 pathwayrelated genes. These included many genes involved in the auxin response, such as auxin response factor 2 (TRINITY_DN56024_c0_g15), auxin-responsive protein IAA27 (TRINITY_DN55735_c1_g8), auxin transporter-like protein 4 (TRINITY_DN53899_c1_g9) and auxin-responsive protein SAUR32 (TRINITY_DN52413_c0_g2). MapMan was used for transcription factor (TF) expression visualization (Fig. 5). Some TFs showed significant upregulation; for example the expression of the TCP (Teosinte branched1/Cycloidea/Proliferating cell factors) family member TCP8 (TRINITY_DN55474_c0_g2) was 4.32 times higher during the P4 period then during the P1 period (P4/P1), and the MADS-box related gene, AGAMOUS-like 42 (TRINITY_DN55218_c1_g4) was 5.29 times higher during the P4 period (P4/P1).

In order to verify the reliability of transcriptomic sequencing results, 10 differentially expressed genes, including TRINITY_DN56024_c0_g15, were selected for qRT-PCR. The results showed that the expression trend of all qRT-PCR results was consistent with the transcriptomic sequencing results, indicating that transcriptomic sequencing results were reliable. 

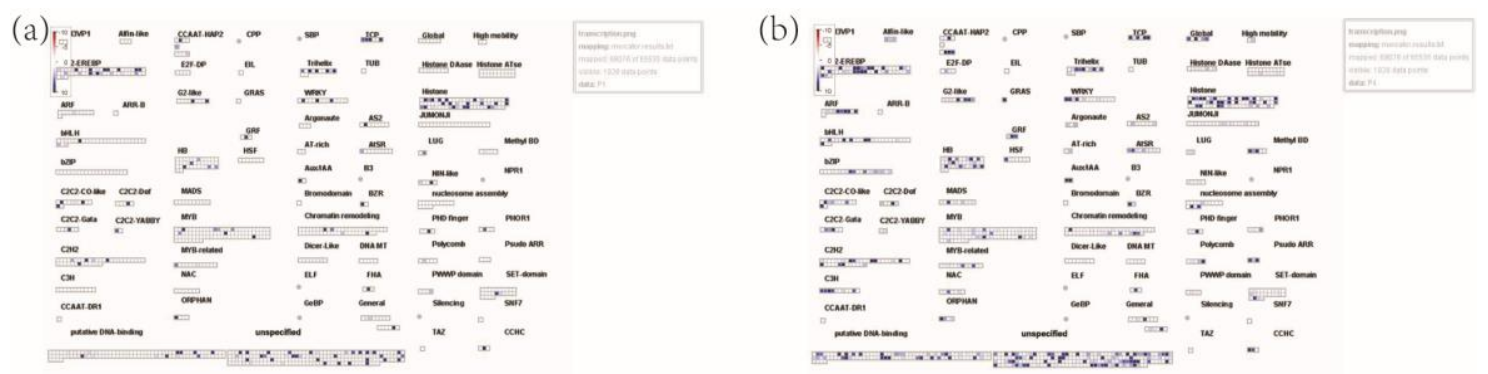

Figure 5. (a) Gene expression of the transcription factor P1 during mapman analysis; (b) Gene expression of the transcription factor P4 during mapman analysis

\section{Discussion}

Apical dominance is the process by which the shoot tip inhibits the growth of the stem bud; the dormancy of the axillary bud is not definitive (Chabikwa et al., 2019). The development of leaf buds into new branches and the growth of leaf buds is precisely controlled according to internal and external factors. These factors include plant age, bud location, available nutrients, light and temperature (Luo et al., 2019). Studies have shown that temperature has the most direct effect on the sprouting of the latent buds of P. massoniana (Zhu et al., 2018). Axillary bud development consists of two stages: the development of meristems in the leaf axils and subsequent growth or dormancy. The branching pattern depends on a key developmental decision: whether the axillary buds grow branches or remain dormant in the leaf axils. The outcome is controlled by environmental and endogenous hormone signaling-mediated stimuli (Domagalska et al.2011; Aguilar-Martinez et al.2007).

The growth of axillary buds can be divided into four stages: initiation and formation of axillary buds, associated inhibition (apical dominance), induction (bud activation) and sustained growth of twin branches (Tan et al., 2019). Zhu et al. (2019a) observed the anatomy of the short-branched buds of $P$. massoniana, they found that the developmental pattern of the short-branched buds was similar to that of the reported long-leaved buds of Pinus. In this study, high-throughput sequencing technology and conventional physiological techniques were used to obtain the transcript expression profiles and the physiological trait data of the four periods: before decapitation, and $10 \mathrm{~d}, 20 \mathrm{~d}$ and $30 \mathrm{~d}$ after the topping (the axillary buds had sprouted after $30 \mathrm{~d}$ ). The physiological data showed that the $\mathrm{N}$ was positively correlated with $\mathrm{K}$ and the correlation index reached 0.6; IAA increased continuously first and then slightly decreased, but ABA decreased continuously, the correlation coefficient of IAA with $\mathrm{ABA}$ was -0.48 . $\mathrm{GA}_{3}$ rises first and then falls, and finally stabilizes. During the axillary bud sprouting process, the (IAA $+\mathrm{GA}_{3}+\mathrm{ZR}$ )/ABA ratio increased gradually from 0.796 before pruning, to 1.746 at the time of bud sprouting (2.19-fold increase).

It has been shown that mechanical damage to the top and axillary bud growth of tobacco (Nicotiana tabacum) may affect ABA transduction, and most ABA-responsive transcripts negatively regulate the sprouting of the axillary buds of tobacco (Wang et al., 2018). This indicates that the increase in $\mathrm{N}$ and IAA contents observed in this study, promoted dormancy release and lateral branching of $P$. massoniana buds. ABA may have a detrimental effect on the dormancy release of the latent buds of $P$. massoniana. In addition, exogenous hormone of 6-BA could promote the dormant bud germination 


$$
-735 \text { - }
$$

earlier and different concentrations of IAA, $\mathrm{GA}_{3}$ and 6-BA could improve the quantity and quality of scions, especially the best treatment is 100ppm GA G $_{3}$ (Zhu et al., 2019b).

TCP proteins directly affect growth through tKEGG multi-group enrichment visualization analysis found that phenylpropanoid biosynthesis (ko00940) and flavonoid biosynthesis (ko00941) were enriched in all three group comparisons. The phenylpropanoid pathway produces a large array of structurally diverse molecules, including hydroxycinnamic acids and flavonoids such as flavonols, anthocyanins, dihydrochalcones and their glycoside derivatives (Dare et al., 2017). Several studies have shown that auxin, cytokinin, strigolactones and other hormones participate in the development of lateral tissues. Auxin maintains apical dominance primarily through polar transport, directly inhibiting cytokinin biosynthesis and inhibiting tissue growth through AXR1-dependent auxin signaling pathways (Tanaka et al., 2006; Tarkowska et al., 2004). Removal of the apical dominance can open the auxin polar transport channels between the axillary bud and the main stem. This allows polar transport of auxin within the main stem to promote the growth of the axillary bud (Balla et al., 2011). The expression of MdPIN15, MdSMXL1, MdSMXL3, MdSMXL4 and MdSMXL11 has been shown to increase with the sprouting and growth of axillary buds. These genes are candidates for the regulation of the sprouting of axillary buds (Liu, 2018). During the sprouting of the latent buds of $P$. massoniana, the IAA content increased continuously and then decreased slightly. Clustering analysis of the significantly differentially expressed genes between the three comparison groups found that most of the metabolic pathways showed a trend of upregulation. The observed IAA transport and responserelated gene activation may be related to axillary bud dormancy release.

The cell cycle, and indirectly affect growth by influencing plant hormone signaling and the circadian clock (Danisman, 2016). Class I TCP genes (TCP6-9, 11, 14-16 and 19-23) are mainly positive regulators of cell division in seed sprouting, leaf and floral organ development, gametophyte development and senescence (Dhaka et al., 2017). Dormancy-associated MADS-box genes are members of the short vegetative phase (SVP)-like MADs-box group and play a key role in the dormancy of perennial plants (Zhao et al., 2018). In this study, the expression of TCP8 (TRINITY_DN55474_c0_g2) was 4.32 times higher during the $\mathrm{P} 4$ period than during P1 (P4/P1), and that of MADSbox related AGAMOUS-like 42 (TRINITY_DN55218_c1_g4) was 5.29 times higher during the $\mathrm{P} 4$ period (P4/P1). The activation of TCP and MADS-box TFs may be associated with the formation of lateral meristems.

\section{Conclusion}

High-throughput sequencing technology and routine physiological experiments were used to obtain the transcript expression profiles and the physiological trait data of four different groups of $P$. massoniana: no-topping, and $10 \mathrm{~d}, 20 \mathrm{~d}$ and $30 \mathrm{~d}$ after topping. There played the crucial roles in the latent bud sprouting of $P$. massoniana of endogenous hormones. Increases in N and IAA content promoted dormancy release and lateral branching of $P$. massoniana buds. ABA had a detrimental effect on the dormancy release of $P$. massoniana. The activation of IAA transport and response-related genes may be associated with the dormancy release of axillary buds. The activation of TCP and MDAS-box transcription factors may be associated with lateral meristem formation.

In this study, the physiological changes and transcript expression of $P$. massoniana during latent bud sprouting were preliminarily revealed, and it was helpful to 
understand the mechanism of latent bud sprouting. In the future study, the change of metabolites during sprouting will be studied, and the clonal utilization of latent bud of P. massoniana will be also carried out.

Acknowledgements. The study was financially supported by the Science and Technology Talent Platform Project of Guizhou Province(grant number [2018]5261),National Key Research and Development Project (grant number 2017YFD0600301) and Guizhou Science and Technology Plan Project (grant number [2017]1098).

\section{REFERENCES}

[1] Aguilar-Martínez, J. A., Poza-Carrión, C., Cubas, P. (2007): Arabidopsis branched acts as an integrator of branching signals within axillary buds. - Plant Cell 19(2): 458-472.

[2] Balla, J., Kalousek, P. (2011): Competitive canalization of pin-dependent auxin flow from axillary buds controls pea bud outgrowth. - The Plant Journal: for Cell and Molecular Biology 65(4): 571-7.

[3] Bolger, A. M., Lohse, M., Usadel, B. (2014): Trimmomatic: a flexible trimmer for Illumina sequence data. - Bioinformatics 30(15): 2114-2120.

[4] Chabikwa, T. G., Brewer, P. B., Beveridge, C. A. (2019): Initial bud outgrowth occurs independent of auxin flow from out of buds. - Plant Physiol 179(1): 55-65.

[5] Chen, X. Y., He, L. H. (1989): A latest way for reproduction of pine - the technique of neeble bundle grafting. - Forestry Research 2(2).

[6] Danisman, S. (2016): TCP transcription factors at the interface between environmental challenges and the plant's growth responses. - Front Plant Sci 7: 1930.

[7] Dare, A. P., Yauk, Y. K., Tomes, S., McGhie, T. K., Rebstock, R. S., Cooney, J. M., Atkinson, R. G. (2017): Silencing a phloretin-specific glycosyltransferase perturbs both general phenylpropanoid biosynthesis and plant development. - Plant J 91(2): 237-250.

[8] Dhaka, N., Bhardwaj, V., Sharma, M. K., Sharma, R. (2017): Evolving tale of TCPs: new paradigms and old lacunae. - Front Plant Sci 8: 479.

[9] Domagalska, M. A., Leyser, O. (2011): Signal integration in the control of shoot branching. - Nat Rev Mol Cell Biol 12(4): 211.

[10] Haas, B. J., Papanicolaou, A., Yassour, M., Grabherr, M., Blood, P. D., Bowden, J. (2013): Denovo transcript sequence reconstruction from RNA-seq using the trinity platform for reference generation and analysis. - Nat Protoc 8(8): 1494.

[11] Langmead, B., Salzberg, S. L. (2012): Fast gapped-read alignment with Bowtie 2. - Nat Methods 9(4): 357.

[12] Liu, X. J. (2018): Genome-Wide Identification, Phylogeny and Expression Analysis of PIN and SMXL Family During Axillary Bud Outgrowth in Apple. - MFA Thesis, Northwest A and F University, Xian.

[13] Luo, L., Takahashi, M., Kameoka, H., Qin, R., Shiga, T., Kanno, Y., Kyozuka, J. (2019): Developmental analysis of the early steps in strigolactone-mediated axillary bud dormancy in rice. - Plant J 97(6): 1006-1021.

[14] Patil, I. (2018): ggstatsplot:"ggplot2" Based Plots with Statistical Details. - CRAN. https://github.com/IndrajeetPatil/ggstatsplot.

[15] Patro, R., Duggal, G., Love, M. I., Irizarry, R. A., Kingsford, C. (2017): Salmon provides fast and bias-aware quantification of transcript expression. - Nat Methods14(4): 417.

[16] Qin, G., Zhou, Z. et al. (2012): Excellent Germplasm Resources of Pinus Massoniana in China. - China Forestry Publishing Press, Beijing, pp. 38-39.

[17] Tan, M., Li, G., Chen, X., Xing, L., Ma, J., Zhang, D., An, N. (2019): Role of cytokinin, strigolactone and auxin export on outgrowth of axillary buds in apple. - Front Recent Dev Plant Sci 10: 616. 


$$
-737-
$$

[18] Tanaka, H., Dhonukshe, P., Brewer, P. B., Friml, J. (2006): Spatiotemporal asymmetric auxin distribution: a means to coordinate plant development. - Cell Mol Life Sci 63(23): 2738-2754.

[19] Tarkowska, D., Tarkowski, P., Göran, S., Astot, C., Anders, N., Norbaek, R. (2004): Auxin regulation of cytokinin biosynthesis in arabidopsis thaliana: a factor of potential importance for auxin-cytokinin-regulated development. - Proc Natl Acad Sci 101(21): 8039-8044.

[20] Wang, X. K. (2006): Plant Physiology and Biochemistry Experiment Principle and Technology. - Higher Education Press, Beijing.

[21] Wang, W. F., Chen, P., Lv, J., Chen, L., Sun, Y. H. (2018): Transcriptomic analysis of topping-induced axillary shoot outgrowth in Nicotiana tabacum. - Gene 646: 169-180.

[22] Xu, C., Chen, X., Chen, Z. (1992): Sampling techniques for larch needle nutrition diagnosis. - Forestry Science and Technology 3: 5-7.

[23] Yu, G., Wang, L. G., Han, Y., He, Q. Y. (2012): Cluster Profiler: an R package for comparing biological themes among gene clusters. - OMICS: J Integr Biol 16(5): 284287.

[24] Zhang, Y., Wei, Z. M., Xi, M., Shi, J. S. (2006): Efficient plant regeneration in vitro in Pinus massoniana. - Journal of Molecular Cell Biology 39(3): 271-276.

[25] Zhao, K., Zhou, Y., Ahmad, S., Xu, Z., Li, Y., Yang, W., Zhang, Q. (2018): Comprehensive cloning of Prunmus mume dormancy associated MADS-Box genes and their response in flower bud development and dormancy. - Front Plant Sci 9: 17.

[26] Zhu, L. H., Wu, X. Q., Qu, H. Y., Ji, J., Ye, J. R. (2010): Micropropagation of Pinus massoniana and mycorrhiza formation in vitro. - PCTOC 102(1): 121-128.

[27] Zhu, Y. Y., Yang, B., Xu, J. J., Zhou, J. W., Wang, G. (2018): Effect of different factors on sprouting of Pinus massoniana dormant buds. - Guizhou Agric Sci 46(8): 32-34.

[28] Zhu, X. K., Wu, F., Shi, Ch., Wang, H., Zhu, Y. (2019a): Morphologic and anatomic observations of Pinus massoniana after axillary bud dormancy release. - Journal of Northeast Forestry University 47(5): 14-18.

[29] Zhu, Y. Y., Xu, J. J., Yang, B., Zhou, J. W., Wang, G. (2019b): Study on the changes of endogenous hormones during the germination of coniferous latent buds of Pinus massoniana. - Guizhou Forestry Sciences and Technology 2: 1-5. 